\title{
Towards the Heisenberg limit in magnetometry with parametric down-converted photons
}

\author{
Aziz Kolkiran and G. S. Agarwal \\ Department of Physics, Oklahoma State University, Stillwater, Oklahoma 74078, USA
}

(Received 28 April 2006; published 16 November 2006)

\begin{abstract}
Recent theoretical and experimental papers have shown how one can achieve Heisenberg-limited measurements by using entangled photons. Here we show how the photons in a noncollinear down-conversion process can be used for improving the sensitivity of magneto-optical rotation by a factor of 4 which takes us towards the Heisenberg limit. Our results apply to sources with arbitrary pumping. We also present several generalizations of earlier results for the collinear geometry. The sensitivity depends on whether the two-photon or four-photon coincidence detection is used.
\end{abstract}

DOI: 10.1103/PhysRevA.74.053810

PACS number(s): 42.50.Dv, 42.25.Kb, 42.25.Lc

\section{INTRODUCTION}

Parametric down-conversion (PDC) is a process that is used to produce light possessing strong quantum features. Photon pairs generated by this process show entanglement with respect to different physical attributes such as time of arrival [1] and states of polarization [2]. They are increasingly being utilized for very basic experiments to test the foundation of quantum mechanics and to do quantum information processing [2-4]. It is also recognized that entangled photon pairs could be useful in many practical applications in precision metrology involving, e.g., interferometry [5-8], imaging [9,10], lithography [11-14], and spectroscopy [15]. There is a proposal [16] to use electromagnetic fields in NOON states to improve the sensitivity of measurements by a factor of $N$. Some implementations of this proposal exist [17]. In particular, the use of photon pairs in interferometers allows phases to be measured to the precision in the Heisenberg limit where uncertainty scales as $1 / N[18]$ as compared to the shot-noise limit where it scales as $1 / \sqrt{N}$. This means that for a large number of particles, a dramatic improvement in measurement resolution should be possible.

In this paper we present an analysis of how parametric down-converted photons could be very useful in getting better spectroscopic information about the medium. We demonstrate how the improvement in magneto-optical rotation (MOR) of light could be realized by employing two different schemes with collinear and noncollinear down-conversion geometries compared to the use of coherent light. We calculate the resolution that can be achieved in the MOR's both by use of coherent light and down-converted light. We discuss the possibility that the Heisenberg limit [19] could be reached in magnetometry by the use down-converted light.

\section{MOR USING COHERENT LIGHT SOURCE}

Consider a single-mode coherent light traveling in the $z$ direction and a linear isotropic medium made anisotropic by application of the magnetic field $\mathbf{B}$ in the $z$ direction. The incident field can be written in the form

$$
\mathbf{E}(z, t)=\exp (-i \omega t+i k z)\left(\hat{\mathbf{x}} \varepsilon_{x}+\hat{\mathbf{y}} \varepsilon_{y}\right)+\text { c.c. }
$$

The medium is described by the frequency- and magneticfield-dependent susceptibilities $\chi_{ \pm}(\omega)$. That means horizon- tally and vertically polarized components of the incident light will rotate on traveling the medium of length $l$ and the field at the exit can be written as

$$
\mathbf{E}(l, t)=\exp (-i \omega t+i k l)\left(\hat{\mathbf{x}} \varepsilon_{x l}+\hat{\mathbf{y}} \varepsilon_{y l}\right)+\text { c.c. }
$$

The rotation of the horizontal and vertical components can be expressed by the relation

$$
\left(\begin{array}{c}
\varepsilon_{x l} \\
\varepsilon_{y l}
\end{array}\right)=R\left(\begin{array}{l}
\varepsilon_{x} \\
\varepsilon_{y}
\end{array}\right),
$$

where

$$
\begin{gathered}
R=e^{i \theta_{+}} e^{i \theta / 2}\left(\begin{array}{cc}
\cos \frac{\theta}{2} & -\sin \frac{\theta}{2} \\
\sin \frac{\theta}{2} & \cos \frac{\theta}{2}
\end{array}\right), \\
\theta=k l\left(\chi_{+}-\chi_{-}\right), \\
\theta_{+}=k l \chi_{+} .
\end{gathered}
$$

The corresponding quantum-mechanical description can be obtained by replacing the classical amplitudes $\varepsilon_{x}$ and $\varepsilon_{y}$ by the annihilation operators $a_{x}$ and $a_{y}$, respectively. For measurements with coherent sources one can look at the intensities of the $x$ and $y$ components of the output when the input is $x$ polarized with coherent-state amplitude $\alpha_{x}$ [see Fig. 1(a)]. Then the measured quantities will be

$$
\begin{aligned}
& I_{x l}=\left\langle a_{x l}^{\dagger} a_{x l}\right\rangle=\left|\alpha_{x}\right|^{2} \cos ^{2} \frac{\theta}{2}, \\
& I_{y l}=\left\langle a_{y l}^{\dagger} a_{y l}\right\rangle=\left|\alpha_{x}\right|^{2} \sin ^{2} \frac{\theta}{2} .
\end{aligned}
$$

One can estimate the minimum detectable rotation angle $\theta_{m}$ by looking at the fluctuations $\Delta N_{d}$ in the photon number difference between horizontal and vertical photons, where the number difference operator is given as $N_{d}$ $=a_{y l}^{\dagger} a_{y l}-a_{x l}^{\dagger} a_{x l}$. This expression is calculated to be $\left(\Delta N_{d}\right)^{2}$ $=\left|\alpha_{x}\right|^{2} \sin ^{2} \theta$, and since the fluctuation noise is 1 , we obtain $\theta_{m} \approx 1 / \sqrt{\langle N\rangle}$ where $\langle N\rangle$ is the mean number of input photons which is equal to $\left|\alpha_{x}\right|^{2}$. 
(a)

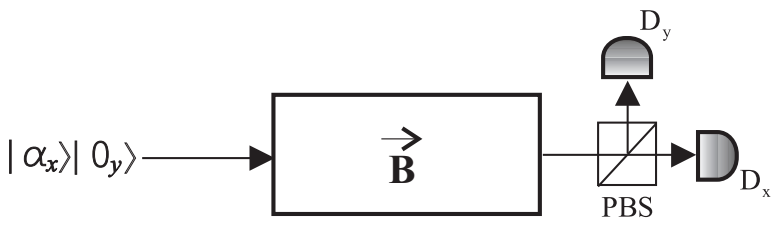

(b)
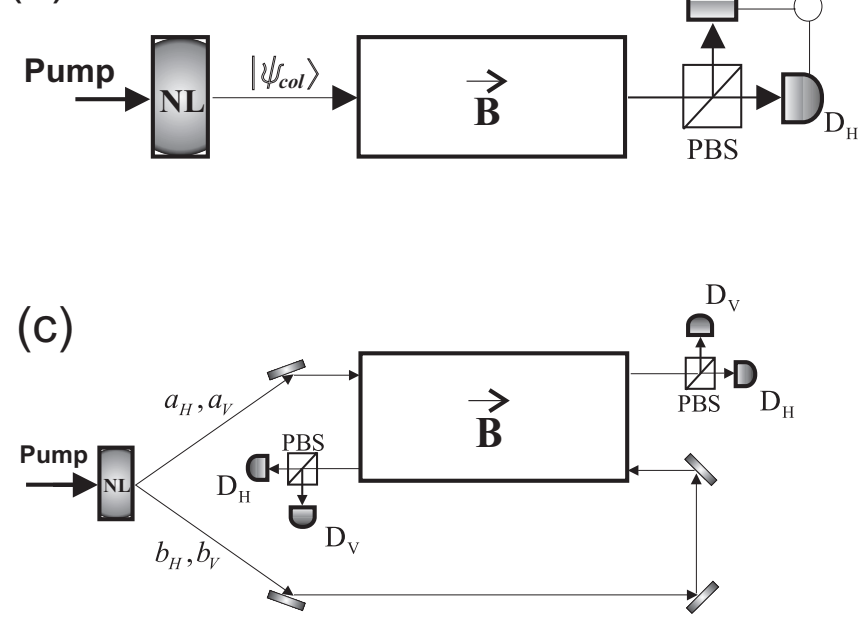

FIG. 1. The setup for the magneto-optical rotation of light by using (a) coherent source, type-II PDC photons with (b) collinear and (c) noncollinear geometry.

\section{MOR USING COLLINEAR TYPE-II PDC AND TWO-PHOTON COINCIDENCE}

We now discuss how the results (7) and (8) are modified if we work with down-converted photons. We first consider the collinear case shown in Fig. 1(b). The state produced in collinear PDC can be written by

$$
\left|\psi_{\text {col }}\right\rangle=\frac{1}{\cosh r} \sum_{n=0}^{\infty}\left(-e^{i \phi} \tanh r\right)^{n}|n\rangle_{H}|n\rangle_{V} .
$$

The values of the parameter $r$ and the phase $\phi$ are related to the pump amplitude of the nonlinear crystal that is used in the down-conversion process and the coupling constant between the electromagnetic field and the crystal. Note that the state $\left|\psi_{\text {col }}\right\rangle$ is a superposition of $n$ photon pairs of horizontally and vertically polarized modes. Inside the medium, these modes rotate with the same rotation matrix $R$ given in Eq. (4)

$$
\left(\begin{array}{l}
a_{H l} \\
a_{V l}
\end{array}\right)=R\left(\begin{array}{c}
a_{H} \\
a_{V}
\end{array}\right) .
$$

One can measure the intensity of each mode:

$$
I_{H} \equiv\left\langle a_{H l}^{\dagger} a_{H l}\right\rangle=\sinh ^{2} r=\left\langle a_{V l}^{\dagger} a_{V l}\right\rangle \equiv I_{V} .
$$

And the two-photon coincidence count is

$$
I_{H V} \equiv\left\langle a_{H l}^{\dagger} a_{V l}^{\dagger} a_{H l} a_{V l}\right\rangle=\cos ^{2} \theta \sinh ^{2} r \cosh ^{2} r+\sinh ^{4} r .
$$

Note the difference between Eqs. (7) and (12) and (13). With collinearly down-converted photons we measure a ro-

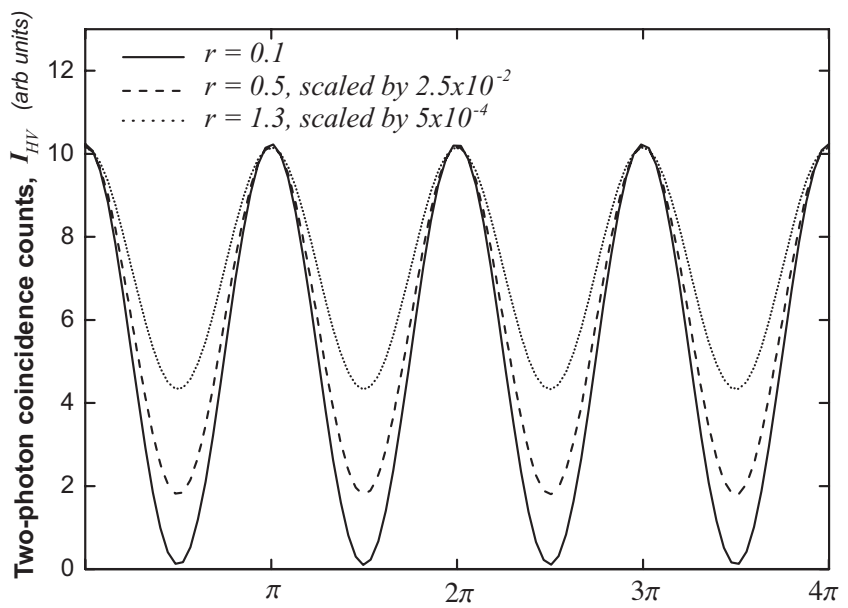

FIG. 2. The MOR plot of two-photon coincidence counts defined by the Eq. (12) in collinear type-II PDC. $r$ is the interaction parameter that defines the pumping strength used in the production of down-converted photons and $\theta=k l\left(\chi_{+}-\chi_{-}\right)$.

tation angle that is twice as large compared with the angle for a coherent input. For $r \ll 1$ we obtain the same result as given in [15]. The fringe pattern and the visibility are given in Figs. 2 and 3. One can calculate the minimum detectable rotation angle again by looking at the fluctuations in the photon number difference $N_{d}$. This is given by $\left(\Delta N_{d}\right)^{2}$ $=4 \sin \mathrm{h}^{2} r \cos \mathrm{h}^{2} \sin ^{2} \theta=(1+\langle N\rangle)\langle N\rangle \sin ^{2} \theta \approx\langle N\rangle^{2} \sin ^{2} \theta$ for large $\langle N\rangle$ where $\langle N\rangle=2 \sinh ^{2} r$. Making $\left(\Delta N_{d}\right) \sim 1$ [19] we get $\theta_{m} \approx 1 /\langle N\rangle$. Note that the sensitivity of this quantity is also improved by a factor of $1 / \sqrt{N}$.

\section{MOR USING NONCOLLINEAR TYPE-II PDC AND FOUR-PHOTON COINCIDENCE}

Next, we discuss the noncollinear PDC case. We have found an arrangement shown in Fig. 1(c) which is especially attractive for improving sensitivity. The entangled photons

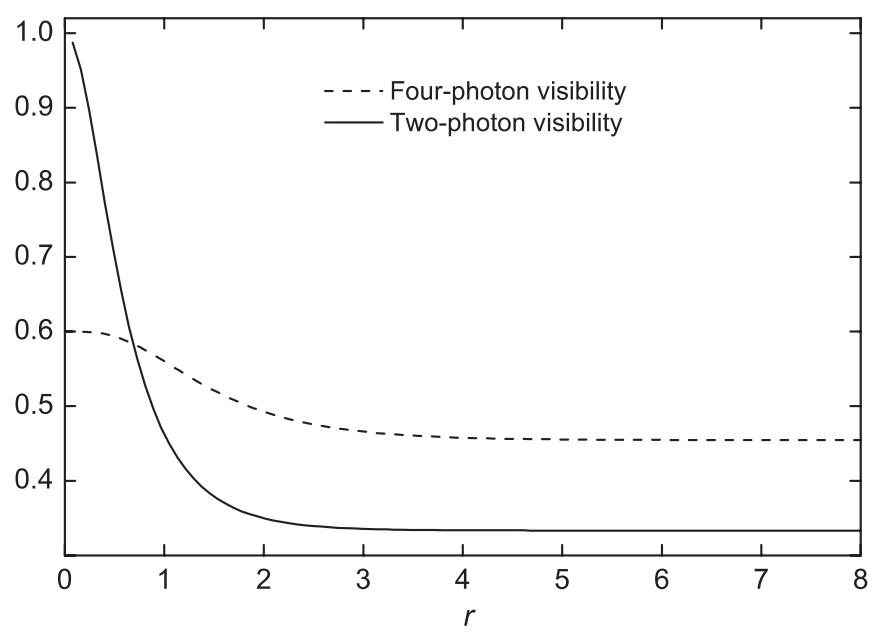

FIG. 3. The visibility of two-photon and four-photon counts defined by Eqs. (12) and (19), respectively. 

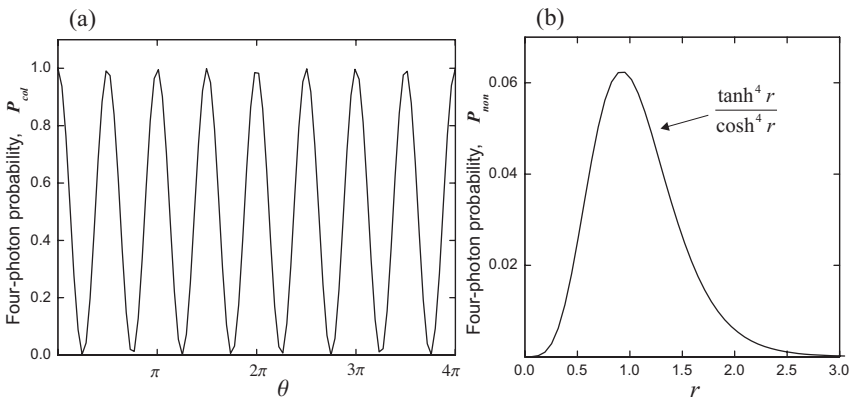

FIG. 4. (a) The normalized four-photon probability defined in Eq. (17) and (b) its envelope with respect to the interaction parameter $r$ in the noncollinear geometry.

are coming in two different spatial modes $a$ and $b$. While one mode (say, $a$ ) is going parallel to $\mathbf{B}$ inside the medium; the other is going antiparallel to it. At the exit we separate the $H$ and $V$ modes by polarizing beam splitters. The state of the input photons can be written in the form [20]

$$
\left|\psi_{n o n}\right\rangle=\frac{1}{\cosh ^{2} r} \sum_{n=0}^{\infty} \sqrt{n+1}(\tanh r)^{n}\left|\psi_{n}\right\rangle,
$$

where

$$
\left|\psi_{n}\right\rangle=\frac{1}{\sqrt{n+1}} \sum_{m=0}^{n}(-1)^{m}|n-m\rangle_{a_{H}}|m\rangle_{a_{V}}|m\rangle_{b_{H}}|n-m\rangle_{b_{V}} .
$$

Here $|m\rangle_{a_{V}}$ represents $m$ vertically polarized photons in mode $a$. Inside the medium, "+" and "-" polarization components of the modes $a$ and $b$ gain phases $k l \chi_{+}$and $k l \chi_{-}$, respectively. Thus we can write an effective Hamiltonian for the evolution of the state $\left|\psi_{\text {non }}\right\rangle$ inside the medium as follows:

$$
H_{\text {med }}=\chi_{+} a_{+}^{\dagger} a_{+}+\chi_{-} a_{-}^{\dagger} a_{-}-\chi_{+} b_{+}^{\dagger} b_{+}-\chi_{-} b_{-}^{\dagger} b_{-},
$$

where

$$
a_{ \pm}=\frac{1}{\sqrt{2}}\left(a_{H} \pm i a_{V}\right), \quad b_{ \pm}=\frac{1}{\sqrt{2}}\left(b_{H} \pm i b_{V}\right) .
$$

The minus sign in front of the $b_{ \pm}$modes comes from the fact that they are traveling antiparallel to the $\mathbf{B}$ field inside the medium. Then one can calculate the probability of detecting four photons in each mode as

$$
\begin{aligned}
P_{\text {non }} & =\left|\left\langle 1_{a_{H}} 1_{a_{V}} 1_{b_{H}} 1_{b_{V}}\left|\exp \left(-i t H_{\text {med }}\right)\right| \psi_{\text {non }}\right\rangle\right|^{2} \\
& =\frac{\tanh ^{4} r}{\cosh ^{4} r} \cos ^{2}(2 \theta),
\end{aligned}
$$

where $t$ is the duration for the state to evolve inside the medium. Note that this four-photon probability has a rotation angle that is 4 times as large compared with the angle for a coherent input. The fringe pattern with respect to $\theta$ and the probability distribution with respect to $r$ are shown in Figs. 4(a) and 4(b).
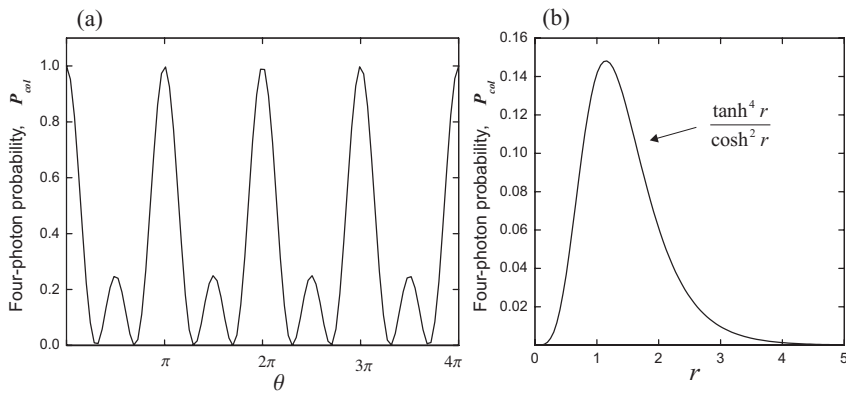

FIG. 5. (a) The normalized four-photon probability defined in Eq. (18) and (b) its envelope with respect to the interaction parameter $r$ at the exit ports of PBS in the collinear geometry.

Next we also examine the four-photon probability in the collinear case. The probability of finding two $H$ photons and two $V$ photons at the exit ports of the polarizing beam splitter is given by

$$
\begin{aligned}
P_{c o l} & =\left|\left\langle 2_{a_{H}{ } a_{V}}\left|\exp \left(-i t H_{m e d}\right)\right| \psi_{c o l}\right\rangle\right|^{2} \\
& =\frac{\tanh ^{4} r}{\cosh ^{2} r} \frac{1}{16}[1+3 \cos (2 \theta)]^{2},
\end{aligned}
$$

where we take $H_{\text {med }}=\chi_{+} a_{+}^{\dagger} a_{+}+\chi_{-} a_{-}^{\dagger} a_{-}$because of the collinear geometry. The normalized plot of this quantity with respect to the magneto-optical rotation angle $\theta$ and the envelope of the probability with respect to $r$ are shown in Figs. 5(a) and 5(b).

On the other hand, one can also calculate the coincidence counts of four photons two-by-two at each detector as given by Glauber's higher-order correlation functions

$$
\begin{aligned}
I_{H H V V}= & \left\langle a_{H l}^{\dagger 2} a_{V l}^{\dagger 2} a_{H l}^{2} a_{V l}^{2}\right\rangle \\
= & \left(3 \cos ^{2} \theta-1\right)^{2} \sinh ^{4} r \cosh ^{4} r \\
& +4\left(3 \cos ^{2} \theta+1\right) \sinh ^{6} r \cosh ^{2} r+4 \sinh ^{8} r .
\end{aligned}
$$

The plot of this quantity for different values of the interaction parameter $r$ and the visibility are shown in Figs. 6 and 3. Note the distinction between Eqs. (18) and (19) which is a reflection of what the detector is set to measure as we explain now. The former is the probability of the state $\left|\psi_{c o l}(t)\right\rangle$ to be projected onto the particular four-photon subspace $|22\rangle-$ i.e.e, $\operatorname{Tr}\left[|22\rangle\langle 22| \rho_{c o l}(t)\right]$ where $\rho_{c o l}(t)=U\left|\psi_{c o l}\right\rangle\left\langle\psi_{c o l}\right| U^{\dagger}$ and $U$ is the unitary operator that represents the evolution of the state by the Hamiltonian $H_{\text {med }}$ in the collinear geometry. On the other hand, coincidence counting of four photons at the detectors $D_{H}$ and $D_{V}$ [see Fig. 1(b)] is represented by the expectation value $\left\langle a_{H}^{\dagger 2} a_{V}^{\dagger 2} a_{H}^{2} a_{V}^{2}\right\rangle=\operatorname{Tr}\left[a_{H}^{\dagger 2} a_{V}^{\dagger 2} a_{H}^{2} a_{V}^{2} \rho_{c o l}(t)\right]$. Note here that the operator $a_{H}^{\dagger 2} a_{V}^{\dagger 2} a_{H}^{2} a_{V}^{2}$ has the spectral decomposition $\sum_{n m}^{\infty} C_{n m}|n m\rangle\langle n m|$ and obviously it contains the projectors of all $(n+m)$-photon subspaces with nonzero coefficients $C_{n m}$. Therefore the four-photon counting process at detectors includes not only $|22\rangle$ but all other states $|n m\rangle$ in $\left|\psi_{c o l}(t)\right\rangle$. Here the state $|n m\rangle$ represents $n$ and $m$ photons in the $a_{H}$ and $a_{V}$ modes, respectively. 


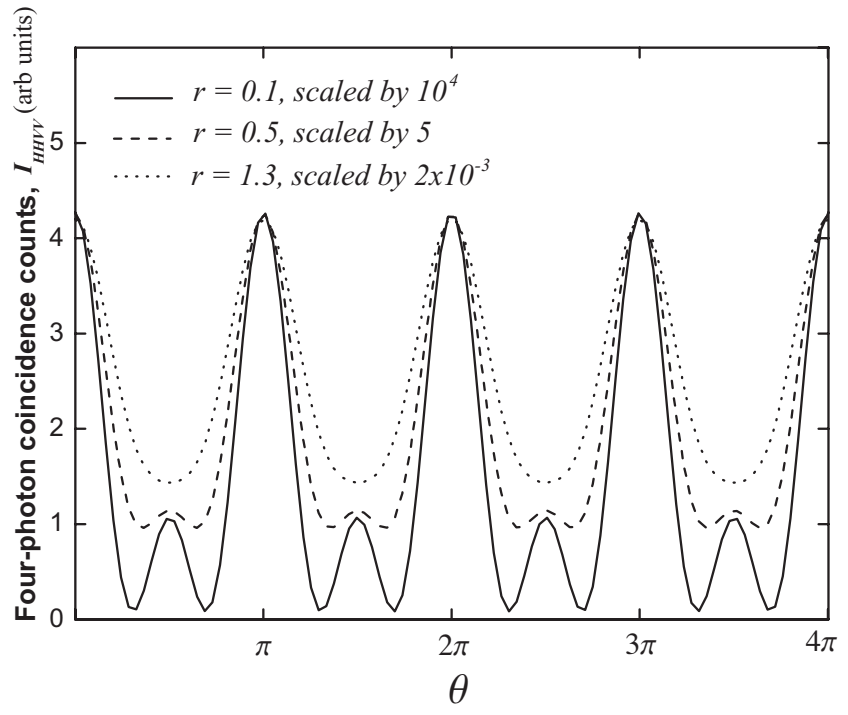

FIG. 6. Four-photon coincidence counts defined in Eq. (19) with different interaction parameter values in the collinear geometry.

\section{CONCLUSION}

We showed that the use of noncollinear type-II PDC light in MOR's increases the sensitivity by a factor of 4 in comparison to coherent light. We note that one can expect to have further flexibility in sensitivity by using suitably prepared atomic samples [21] as earlier studies [22-24] have shown how the sensitivity of interferometers can be improved by the use of entangled atoms.

We also give an argument that minimum rotation uncertainty scales to the Heisenberg limit by the use of downconverted photons. It should be noted that the Heisenberg limit should be understood as an approximate limit at a large mean photon number; that is, the rotation uncertainty approaches the order of $1 /\langle N\rangle$ for large $\langle N\rangle$ [8]. The regime with an interaction parameter value of $r=1.3$ has already been reached in the experiment [25] giving entanglement of 12 photons and evidence also was given for entanglement up to 100 photons.

\section{APPENDIX: FOUR-PHOTON PROBABILITY}

In this appendix we show the details of the calculation leading to the result given in Eq. (17). One can obtain the result first by solving the Schrödinger equation for the state $\left|1_{a_{H}} 1_{a_{V}} 1_{b_{H}} 1_{b_{V}}\right\rangle$ in the four-photon subspace of the electromagnetic field and having the inner product with the state $|\psi\rangle_{\text {non. }}$. Since the parts of the Hamiltonian having $a$ and $b$ modes commute, we can solve the Schrödinger equation for the states $\left|1_{a_{H}} 1_{a_{V}}\right\rangle$ and $\left|1_{b_{H}} 1_{b_{V}}\right\rangle$ separately. Let us start with a general time-dependent state in the $a_{H}$ and $a_{V}$ modes which contain two photons totally:

$$
|\phi(t)\rangle=c(t)|20\rangle+d(t)|02\rangle+f(t)|11\rangle,
$$

with the initial condition $|\phi(0)\rangle=|11\rangle$. Solving the Schrödinger equation by using the effective Hamiltonian $H$ $=\chi_{+} a_{+}^{\dagger} a_{+}+\chi_{-} a_{-}^{\dagger} a_{-}$gives us the result

$$
\begin{aligned}
|\phi(t)\rangle= & e^{-i t \chi}\left[\frac{1}{\sqrt{2}} \sin (\Omega t)|20\rangle-\frac{1}{\sqrt{2}} \sin (\Omega t)|02\rangle\right. \\
& +\cos (\Omega t)|11\rangle],
\end{aligned}
$$

where $\chi=\chi_{+}+\chi_{-}$and $\Omega=\chi_{+}-\chi_{-}$. For a medium of length $l$, the angle $\Omega t$ corresponds to the MOR angle $\theta$ which is given in Eq. (5). The solution for the state $\left|1_{b_{H}} 1_{b_{V}}\right\rangle$ can be obtained just by replacing $\theta$ by $-\theta$ because the direction of propagation of the $b$ modes is opposite to that of $a$ modes inside the medium. This is the reason that the part of the effective Hamiltonian for the $b_{ \pm}$modes takes a minus sign in Eq. (15). Consequently we can write the solution of the Schrödinger equation for the state $\left|1_{a_{H}} 1_{a_{V}} 1_{b_{H}} 1_{b_{V}}\right\rangle$ as

$$
\begin{aligned}
& \exp \left(-i t H_{\text {medium }}\right)\left|1_{a_{H}} 1_{a_{V}} 1_{b_{H}} 1_{b_{V}}\right\rangle \\
& =\exp (-i t \chi)\left[\frac{1}{\sqrt{2}} \sin \theta|20\rangle-\frac{1}{\sqrt{2}} \sin \theta|02\rangle+\cos \theta|11\rangle\right] \\
& \otimes \exp (-i t \chi)\left[-\frac{1}{\sqrt{2}} \sin \theta|20\rangle\right. \\
& \left.+\frac{1}{\sqrt{2}} \sin \theta|02\rangle+\cos \theta|11\rangle\right]
\end{aligned}
$$

Taking the inner product of this with the state $|\psi\rangle_{\text {non }}$ and having the absolute square gives us the result given in Eq. (17).

The result given in Eq. (18) can be obtained by following the same method given above.
[1] S. Friberg, C. K. Hong, and L. Mandel, Phys. Rev. Lett. 54, 2011 (1985).

[2] P. G. Kwiat, K. Mattle, H. Weinfurter, A. Zeilinger, A. V. Sergienko, and Y. Shih, Phys. Rev. Lett. 75, 4337 (1995).

[3] L. Mandel and E. Wolf, Coherence and Quantum Optics (Cambridge University Press, Cambridge, England, 1995), Chaps. 12 and 22.

[4] A. Zeilinger, Rev. Mod. Phys. 71, S288 (1999).

[5] C. M. Caves Phys. Rev. D 23, 1693 (1981).

[6] B. Yurke, S. L. McCall, and J. R. Klauder, Phys. Rev. A 33,
4033 (1986).

[7] J. P. Dowling, Phys. Rev. A 57, 4736 (1998).

[8] M. J. Holland and K. Burnett, Phys. Rev. Lett. 71, 1355 (1993).

[9] T. B. Pittman, Y. H. Shih, D. V. Strekalov, and A. V. Sergienko, Phys. Rev. A 52, R3429 (1995).

[10] A. F. Abouraddy, B. E. A. Saleh, A. V. Sergienko, and M. C. Teich, Phys. Rev. Lett. 87, 123602 (2001).

[11] A. N. Boto, P. Kok, D. S. Abrams, S. L. Braunstein, C. P. Williams, and J. P. Dowling, Phys. Rev. Lett. 85, 2733 (2000). 
[12] G. S. Agarwal, R. W. Boyd, E. M. Nagasako, and S. J. Bentley, Phys. Rev. Lett. 86, 1389 (2001).

[13] G. Björk, L. L. Sanchez-Soto, and J. Söderholm, Phys. Rev. Lett. 86, 4516 (2001).

[14] M. D’Angelo, M. V. Chekhova, and Y. Shih, Phys. Rev. Lett. 87, 013602 (2001).

[15] G. S. Agarwal and M. O. Scully, Opt. Lett. 28, 462 (2003).

[16] H. Lee, P. Kok, N. J. Cerf, and J. P. Dowling, Phys. Rev. A 65, 030101(R) (2002).

[17] P. Walther et al., Nature (London) 429, 158 (2004); M. W. Mitchell, J. S. Lundeen and A. M. Steinberg, ibid. 429, 161 (2004).
[18] V. Giovannetti, S. Lloyd, and L. Maccone, Science 306, 1330 (2004).

[19] Z. Y. Ou, Phys. Rev. A 55, 2598 (1997).

[20] P. Kok and S. L. Braunstein, Phys. Rev. A 61, 042304 (2000).

[21] B. Julsgaard, A. Kozhekin, and E. S. Polzik, Nature (London) 413, 400 (2001).

[22] B. Yurke, Phys. Rev. Lett. 56, 1515 (1986).

[23] G. S. Agarwal and R. R. Puri, Phys. Rev. A 49, 4968 (1994).

[24] D. J. Wineland, J. J. Bollinger, W. M. Itano, F. L. Moore, and D. J. Heinzen, Phys. Rev. A 46, R6797 (1992).

[25] H. S. Eisenberg, G. Khoury, G. Durkin, C. Simon, and D. Bouwmeester, Phys. Rev. Lett. 93, 193901 (2004). 